\title{
Deduction of time-dependent machine tool characteristics by fuzzy-clustering
}

\author{
${\text { Uwe Frie } \beta^{1 *} \text {, Martin Kolouch }}^{1}$ and Matthias Putz \\ ${ }^{I}$ Fraunhofer Institute for Machine Tools and Forming Technology IWU, Chemnitz, Germany \\ ${ }^{*}$ Corresponding author. Tel.: +49-371-5397-1393; fax: +49-371-5397-6-1393; \\ E-mail address: uwe.friess@iwu.fraunhofer.de
}

\begin{abstract}
With the onset of ICT and big data capabilities, the physical asset and data computation is integrated in manufacturing through Cyber Physical Systems (CPS). This strategy also denoted as Industry 4.0 will improve any kind of monitoring for maintenance and production planning purposes. So-called bigdata approaches try to use the extensive amounts of diffuse and distributed data in production systems for monitoring based on artificial neural networks (ANN). These machine learning approaches are robust and accurate if the data base for a given process is sufficient and the scope of the target functions is curtailed. However, a considerable proportion of high-performance manufacturing is characterized by permanently changing process, workpiece and machine configuration conditions, e.g. machining of large workpieces is often performed in batch sizes of one or of a few parts. Therefore, it is not possible to implement a robust condition monitoring based on ANN without structured data-analyses considering different machine states - e.g. a certain machining operation for a certain machine configuration. Fuzzy-clustering of machine states over time creates a stable pool representing different typical machine configuration clusters. The time-depending adjustment and automatized creation of clusters enables monitoring and interpretation of machine tool characteristics independently of single machine states and pre-defined processes.
\end{abstract}

Keywords: Fuzzy logic, Machine tool, Machine learning, Clustering.

\section{Introduction}

Technological value adding by extracting of CPS-capabilities is acting as selective pressure not only at academicals levels but already on the shop floor [1-3]. Integrally modules are predictive maintenance and cloud-based monitoring of production systems [4-6]. In [7] and [8] the authors introduced an approach to overcome limits in condition monitoring of large and special-purpose machine tools. The core challenge to address is the time-based change in nearly every internal and external constrainparameter (Fig. 1). 


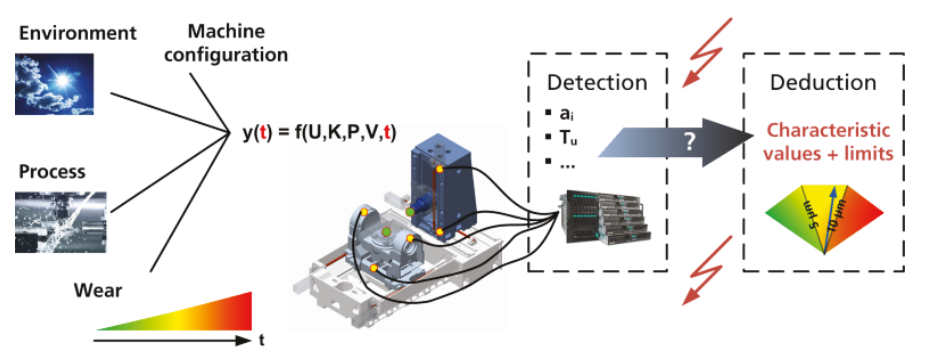

Fig. 1. Challenges in deduction of limits based on measuring data

This results in difficulties to correlate any kind of measuring data with the health state of the machine and its components. Measures to address these challenges are:

1. Definition of Machine States (MSs) based on trigger parameters (TPs) (Table 1).

2. Deduction and comparison of Characteristic Values (CVs) is only carried out

a. for the same machine state

b. Gradually for a cluster resulting from the fuzzy-clustering (see 5 below)

3. Deduction of dynamic limits for the CVs over time

4. Fuzzy-based interpretation of the current $\mathrm{CV}$-values regarding their expectation values (see section 5, Fig. 5)

5. Fuzzy-Clustering of MSs to create a stable pool including a broad range of characteristically configurations of the machine tool

\subsection{Limits of cluster analyses based on pre-defined machine states}

The fuzzy clustering of pre-defined MSs can be adequate for monitoring of components with clear objectives, e.g. the health state. Essential basis is a balanced definition of MSs by a maintenance expert. Therefore the pre-definition of MSs is prone to an unexperienced workforce. More challenging is the altering of processes and workpiece batches which leads to a decay of the initial defined MSs. The expert therefore needs to define new relevant MSs and exclude old ones from the "pool" (see Fig. 9 in [8]).

Further potentials can be obtained if the pre-definition of MSs is replaced by an auto-derivation of MSs and a subsequent fuzzy clustering of these MSs with the objective of a broad characterization of the machine tool configurations over time. For this purpose, a tree-step machine-learning cycle is introduced subsequently and described in the following sections:

1. Auto-definition of MS by segmentation of MS parameters (section 2)

2. Deriving of Characteristic Values (CVs) for every state as described in [8]

3. MS-TP-reduction: Correlation analyses between MSs, CVs, parameter reduction and exclusion of non-significant MSs (section 3 and 4)

4. Fuzzy-clustering of MSs including derivation of Cluster-CVs (section 5)

5. Deriving of machine-characterizing Clusters which represent concrete categories of machine tools, e.g. heavy machining for certain feed axes configuration. 


\section{Auto-definition of MSs by segmentation of TPs for different parameter numbers}

A typical pre-defined MS is characterized by a subset of TPs as presented in [7] (Table 1). The MSs depict in Table 1 are represented by using different TPs for an axis stroke (see Fig. 2).

Table 1. Normalized data of MSs using the relative normalization of TP, overall cycle.

\begin{tabular}{lccccccccc}
\hline MS & $\mathbf{1}$ & $\mathbf{2}$ & $\mathbf{3}$ & $\mathbf{4}$ & $\mathbf{5}$ & $\mathbf{6}$ & $\mathbf{7}$ & $\mathbf{8}$ & $\mathbf{9}$ \\
TP & & & & & & & & & \\
\hline 1.1 Automatic mode & 1 & 1 & 1 & 1 & 1 & 1 & 1 & 1 & 1 \\
3.1 x-pos. & 1 & 1 & 1 & 1 & 1 & 1 & 1 & 1 & 1 \\
4.1 y-pos. & 0.5 & 0.5 & 0.5 & 0.5 & 0.5 & 0.5 & 0.5 & 0.5 & 0.5 \\
4.2 y-pos. $(\Delta)$ & 0 & 0 & 0 & 0 & 0 & 0 & 0 & 0 & 0 \\
5.1 z-pos. & 1 & 0.5 & 0 & 0.86 & 0.41 & 0.14 & 0.05 & 0.55 & 0.95 \\
6.1 Jerk & 1 & 1 & 1 & 1 & 1 & 1 & 1 & 1 & 1 \\
7.1 Acceleration & 1 & 1 & 1 & 0.5 & 0.5 & 0 & 0.75 & 0.75 & 0.75 \\
8.1 Feed rapid traverse & 1 & 1 & 1 & 0 & 0.67 & 0.83 & 0.67 & 1 & 1 \\
9.1 Temperature of y2 & 0 & 0.40 & 0.66 & 0.81 & 0.96 & 0.91 & 1 & 0.71 & 0.70 \\
ball-screw nut & & & & & & & & & \\
\hline
\end{tabular}

TPs can vary in a broad range, e.g. the current position of an axis or the feed. A combination that doesn't occur in praxis - e.g. a stroke between 0 and $1 \mathrm{~mm}$ for a given axis - is not detectable and therefore it does not increase the complexity. However an axis stroke of $1000 \mathrm{~mm}$ could be divided from any numerical integer between 2 and $\infty$ in principle. Thus it is still necessary to have an upfront definition of TPs ranges. A practical solution for dynamic TPs like the jerk, the acceleration or the feed consists in definition of altering-constrains to intersect a MS in sub-phases.

A MS is not a singular event but a process which is characterized by a given timespan. Real-life processes of machine tools are continuous and can be fragmented in several sub-phases by various measures. An example would be a boring operation with a specific tool. Another one could be the stroke of a single axis as depicted in Table 2 and Fig. 2.

The definition of an overall process is complex and may vary depending on the desired application or monitoring object. This process would be the highest level of a MS as depict in Table 1. The y-axis executes a stroke from $300 \mathrm{~mm}$ up to $2400 \mathrm{~mm}$ and back, therefor representing a complete cycle. This overall stroke can consequently be divided into several sup-phases which can be treated as discrete MS. These "subMS" can be identified in dependence of the altering of dynamic parameters as described in Table 2. To distinguish them from each other every sub-MS is described by numerical values depending on the level of the dynamic parameter (Table 2, left). Alternative identifications are also conceivable. However the introduced description based on levels links physical parameters directly to the sub-MSs. 
Table 2. Levels of MSs in dependence of the dynamic y-axis stroke.

\begin{tabular}{|c|c|c|c|c|c|c|c|c|c|}
\hline \multicolumn{7}{|c|}{ Level } & Description (numbers in [mm]) & $\begin{array}{c}\text { Length } \\
{[\mathrm{mm}]}\end{array}$ & $\begin{array}{c}\text { Number of } \\
\text { MS per level }\end{array}$ \\
\hline 0 & 0 & 0 & 0 & 0 & 0 & 0 & Overall stroke & $2 \times 2100$ & 1 \\
\hline 1 & 0 & 0 & 0 & 0 & 0 & 0 & Forward stroke (FS) & 2100 & \\
\hline 2 & 0 & 0 & 0 & 0 & 0 & 0 & Backward stroke (BS) & 2100 & 2 \\
\hline 1 & 1 & 1 & 0 & 0 & 0 & 0 & FS, dynamic phase (DP), 300-500 & 200 & \\
\hline 1 & 1 & 2 & 0 & 0 & 0 & 0 & FS, DP, $1250-1450$ & 200 & \\
\hline 1 & 1 & 3 & 0 & 0 & 0 & 0 & FS, DP, 2200-2400 & 200 & \\
\hline 1 & 2 & 1 & 0 & 0 & 0 & 0 & FS, positioning (PO), 500-1250 & 750 & 1 \\
\hline 1 & 2 & 2 & 0 & 0 & 0 & 0 & FS, PO, $1450-2200$ & 750 & 10 \\
\hline 2 & 1 & 1 & 0 & 0 & 0 & 0 & BS, DP, 2400-2200 & 200 & \\
\hline$\ldots$ & $\ldots$ & ... & $\ldots$ & $\ldots$ & $\ldots$ & $\ldots$ & $\ldots$ & $\ldots$ & \\
\hline 2 & 2 & 2 & 0 & 0 & 0 & 0 & BS, PO, $1250-500$ & 750 & \\
\hline 1 & 1 & 1 & 1 & 1 & 0 & 0 & FS, DP, acceleration (AC), 300-( )375 & 75 & \\
\hline 1 & 1 & 2 & 1 & 2 & 0 & 0 & FS, DP, AC, 1250-( )1325 & 75 & \\
\hline$\ldots$ & $\ldots$ & ... & $\ldots$ & $\ldots$ & $\ldots$ & $\cdots$ & $\ldots$ & $\ldots$ & 30 \\
\hline 1 & 1 & 1 & 2 & 1 & 0 & 0 & FS, DP, constant feed (CF), $(\sim) 375-(\sim) 425$ & 170 & \\
\hline$\ldots$ & $\ldots$ & ... & $\ldots$ & $\ldots$ & $\ldots$ & ... & $\ldots$ & $\ldots$ & \\
\hline 1 & 1 & 1 & 1 & 1 & 1 & 1 & FS, DP, AC, positive jerk (PJ), 300-( )304 & 3,33 (theor.) & 50 \\
\hline
\end{tabular}

If the lowest possible level is defined by the direction of the jerk, a maximum of 50 sub-phases can be identified based on path dynamics. We divide the overall stroke in 12 sub-phases based on the identification levels 1-3 of Table 2 for demonstration purposes as depicted in Fig. 2. Practically other TPs like the dynamic path of a second axis as well as process parameters could also vary in parallel.

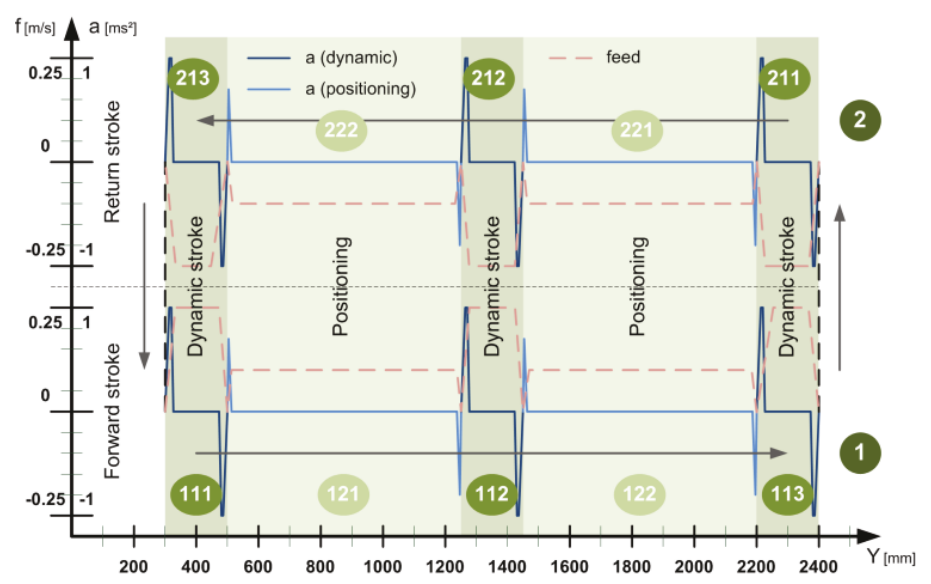

Fig. 2. Test cycle used in [8] including sub-phases of MSs 
Obviously the auto-detection of any possible MS based on time-dependent changes of any considered TP is not a practicable solution. Therefore a parallelization approach is suggested, where MPs based on different TPs for different sub-phases down until the level where the TPs still vary - are created, CVs derived and correlation analyses between MSs and TPs carried out. This overall approach is depicted in Fig. 3.

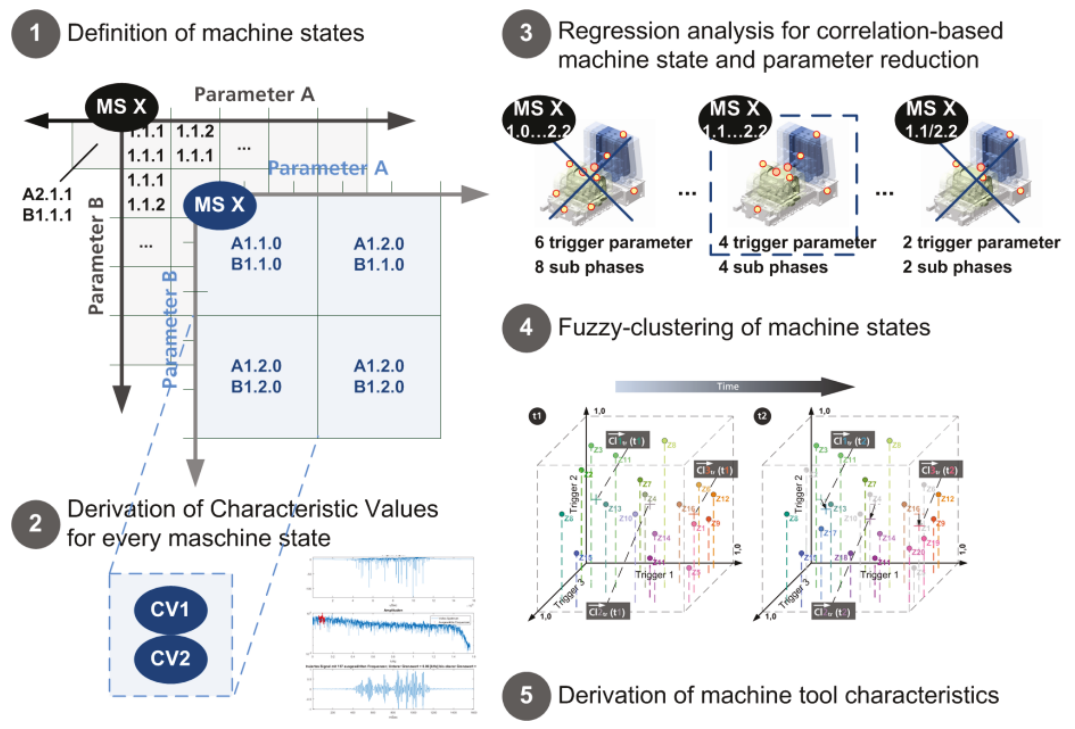

Fig. 3. Suggested approach for automatic MS- and TP reduction

\section{Regression analysis for correlation-based machine state and parameter reduction}

The fuzzy clustering of MSs, as presented in [8] can be exercised without any consideration of possible correlations between TPs and CVs. This is possible for a limited number of pre-defined MSs based on practical considerations about components of interest and - heuristically anticipated - correlations between CVs and TPs. If a broad range of TPs is combined with a variable resolution of TP sections as well as time spans the clustering of all combinations - for every $\mathrm{CV}$ - becomes unpractical, statistically challenging and the information content decays. Therefore a reduction of significant MS and TPs for these states is necessary. This task can be addressed by the usage of an artificial neural network (ANN), but the robustness and accuracy of such depends heavily on the quantity of training data. This means that every relevant MS has to occur several times before the ANN can play off its strength. This is not a given in non-serial machine tool applications as described in section 1.

For this purpose, regression analysis between the TPs and the CVs can be employed as suggested in this paper. Based on the introduced cycle, a regression analysis was carried out. The input variables (TPs) and the responses (CVs) used in the regres- 
sion analysis are shown in Table 8. This includes all varying parameters of the MS. The considered MS regression analysis does not aim to a quantification of the regression function between the input variables and the responses but it should statistical validate the significance of the input variables (for more detail see [9]). Thus, a linear function without any interactions is chosen for the regression analysis.

Table 3. Defined input variables and responses in the regression analysis

\begin{tabular}{lc}
\hline \multicolumn{1}{c}{ Input variables $=$ TPs } & Responses $=\mathbf{C V s}$ \\
\hline z-position & Effective vibration level \\
Acceleration & Frequency of the highest peak \\
Feed rapid traverse & \\
Temperature of the ball-screw nut & \\
\hline
\end{tabular}

The included MSs are 10 sub-phases of Fig. 2 for every TP-combination of Table 1. Sup-phases 113 and 213 (Fig. 2) are not considered due to their corrupted measurement data. It should be noted the TPs 4.1 and 4.2 vary in accordance to the subphases. Therefore 90 different - but related - MS are taken into account.

\section{$4 \quad$ Practical example}

The test cycle of Fig. 2 was derived for the 9 MS in Table 1 (Fig. 4). 51 cycles were successively executed for each MS, resulting in an overall time of 2550 s. Every cycle includes all sub-phase ("sub-MS") of Fig. 2.
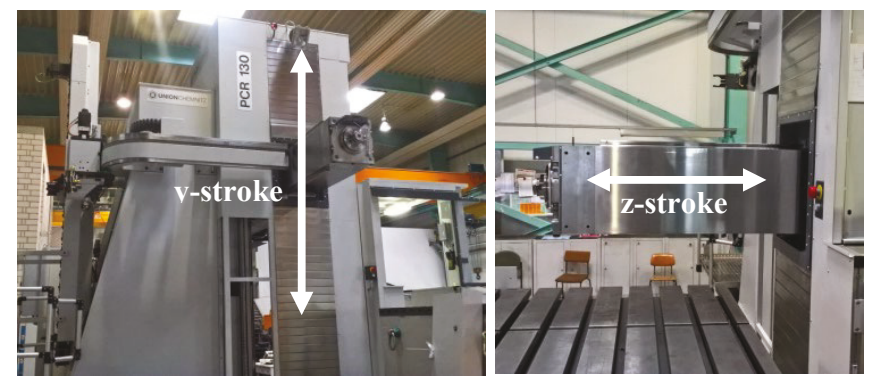

Fig. 4. UNION PCR130 machine; y-and z-axis used for the test cycles

Based on these cycles, a linear regression analyses was derived for the sub-phases using the commercial software Cornerstone ${ }^{\circledR}$. The aim of the regression analyses is not to derive a quantitative model with the aim to predict the CVs based on the TPs. The data available is not sufficient for such a purpose. The regression model is only linear and not representative for the TPs as well as the CVs overall range. However, the regression analysis deducts significance terms for every input-parameter (= TP), therefore distinguishing the relevant TPs for a given CV (responses in Table 4) from the irrelevant ones. Furthermore, when comparing the significance terms of the TPs with the adjusted R-Square value of the correlation analysis we obtain an assessment 
to define adequate sub-phases. Additionally the correlation between the significant TPs (Covariance matrix) is checked to exclude TPs with high covariance's. For example the temperature has an even higher significance-term in sub-phase 112 than the feed. However the Covariance matrix indicates that the temperature is highly correlated to the Temperature $(-0,9861)$ and should therefor excluded for the subsequent clustering for the CV fmax. Successively the number of relevant MSs is significantly reduced. The number of relevant TPs is simultaneously reduced. Table 4 depicts the overall result for all 10 sub-phases and 4 inputs, carried out separately for each of the 9 MSs from Table 1.

Table 4. Correlation analysis results for the sub phases of MS 1-9 and both CVs.

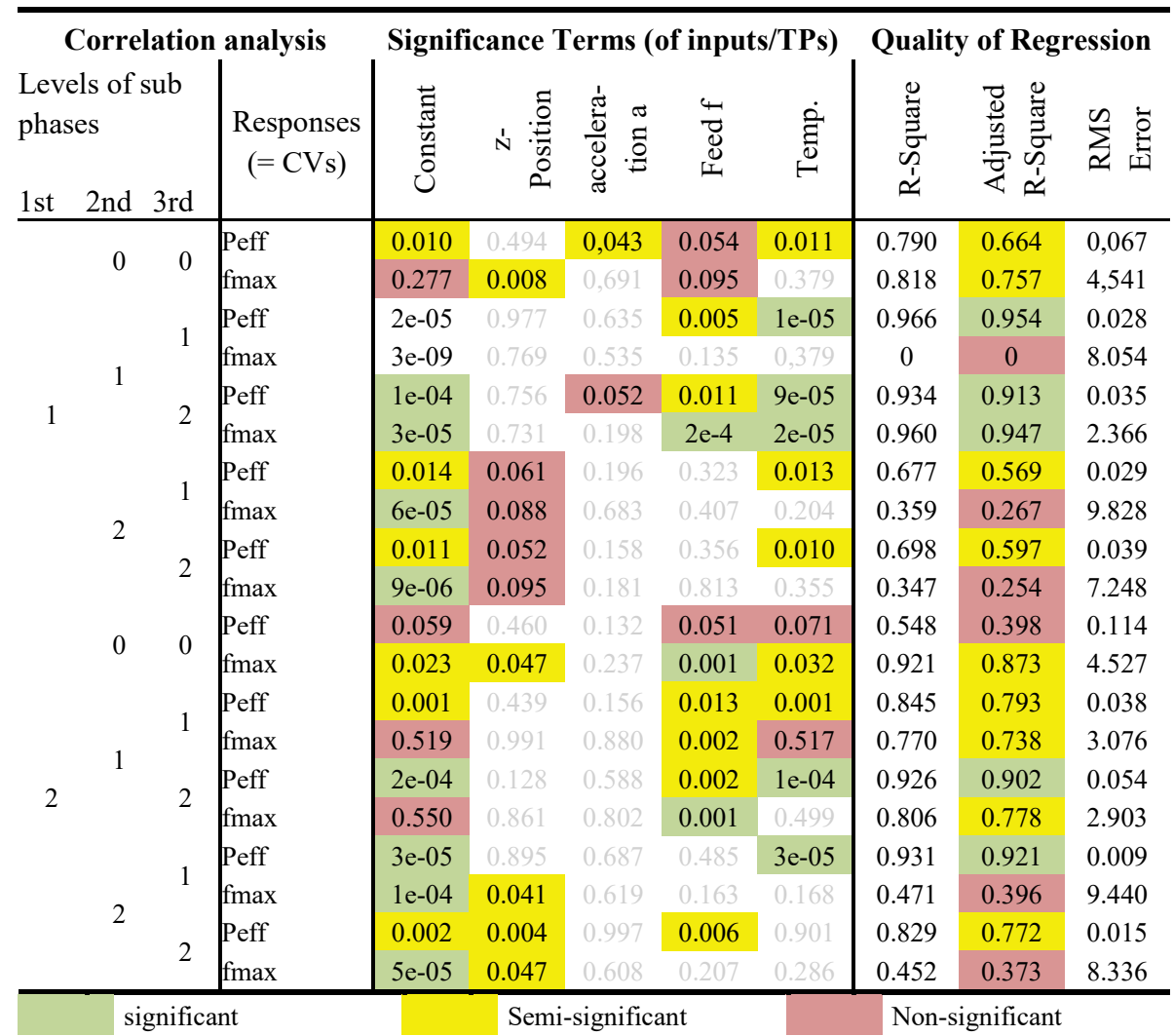

Several important conclusions can be detracted from the results of the correlation analysis and the subsequent survey of Covariance matrix of the significant TPs:

- The most promising sub-phases with the best correlations are the dynamic phases in the middle of the axis stroke; the auto-definition detects this sub-phase MSs

- The effective vibration level is clearly correlated to the temperature of the nut

- The ball pass frequency of the ball-screw nut outer ring is clearly correlated to the feed (the frequency can be calculated based on geometric parameters) 
- The quality of regression for the effective Vibration level (Peff) is significant in more sub-phases and therefore more generally usable than the ball pass frequency of the ball-screw (Y2)nut (fmax)

Therefore the auto detection mechanism would choose sub-phases 112 and 212 as most relevant for monitoring. In regard to the CVs, the temperature remains the only relevant TP for the effective Vibration level while the feed remains the only relevant TP for the outer ring frequency of the ball-screw nut.

\section{Deduction of machine characteristics based on clustering}

The clustering was deducted solely on base of the two relevant TPs for each of the two CVs as described in section 4. The algorithm is described in detail in [8] based on [9]. Every MS is gradually attributed to the cluster centres. The relevant TP 8.1 and 9.1 do not vary in accordance to the sub-phases, so the clustering solely depends on the (average) TP of the 9 MS. We obtain cluster centres at $0.71 / 0.99 / 0.00$ for TP 8.1 (feed rapid traverse) respectively $0.09 / 0.92 / 0.64$ for TP 9.1 (temperature of y2 ball screw nut). Table 5 depicts the TP-values for each MS and their affiliation rate.

Table 5. Normalized TP and affiliation rates per cluster for all MS; optimization cycle $\mathrm{n}_{\mathrm{opt}}=$ 100 ; fuzzifier $w=1.5$

\begin{tabular}{|c|c|c|c|c|c|c|c|c|c|c|}
\hline \multicolumn{2}{|c|}{ Maschine states } & \multirow{2}{*}{1} & \multirow{2}{*}{2} & \multirow{2}{*}{3} & \multirow{2}{*}{4} & \multirow{2}{*}{5} & \multirow{2}{*}{6} & \multirow{2}{*}{7} & \multirow{2}{*}{8} & \multirow{2}{*}{9} \\
\hline \multicolumn{2}{|c|}{ Relevant TPs } & & & & & & & & & \\
\hline \multirow{2}{*}{\multicolumn{2}{|c|}{$\begin{array}{l}\text { 8.1 Feed rapid traverse (for } \\
\text { CV2) } \\
9.1 \text { Temperature of y2 ball- } \\
\text { screw nut (for CV1) }\end{array}$}} & 1 & 1 & 1 & 0 & 0.67 & 0.83 & 0.67 & 1 & 1 \\
\hline & & 0 & 0.40 & 0.66 & 0.81 & 0.96 & 0.91 & 1 & 0.71 & 0.70 \\
\hline \multicolumn{2}{|c|}{ Cluster } & \multicolumn{9}{|c|}{ Affiliation rates per cluster } \\
\hline \multirow{2}{*}{1} & ТР 8.1 & 1 & 0 & 0 & 0 & 1 & 0.732 & 1 & 0 & 0 \\
\hline & ТР 9.1 & 1 & 0.273 & 0 & 0 & 0 & 0 & 0 & 0 & 0 \\
\hline \multirow[b]{2}{*}{ 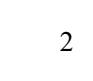 } & ТP 8.1 & 0 & 1 & 1 & 0 & 0 & 0.268 & 0 & 1 & 1 \\
\hline & ТР 9.1 & 0 & 0.034 & 0 & 0.857 & 1 & 1 & 0.997 & 0.013 & 0.06 \\
\hline \multirow[b]{2}{*}{ 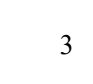 } & ТР 8.1 & 0 & 0 & 0 & 1 & 0 & 0.000 & 0 & 0 & 0 \\
\hline & ТР 9.1 & 0 & 0.693 & 1 & 0.143 & 0 & 0 & 0.003 & 0.987 & 0.994 \\
\hline
\end{tabular}

Based on the affiliation rates of each MS the clusters represent typical CV-progressions as depicted in Fig. 5 for CV1 (effective vibration level). We obtain several alarms for cluster 1 (Fig. 5 left) with limits corresponding to a band in the +/$3 \sigma$ range. This is due to the fact that cluster 1 represents the head-up of the machine tool representing an unsettled pool of MSs (essentially MS 1). Alternatively a band of $+/-6 \sigma$ for limit calculation can be used.

The auto-reduction of relevant TP and MS generates clusters which represent typical conditions of a machine tool. When combined with CV-information's and by subsequent structure-attribution the gathering of machine tool characteristics over time is achievable. 
A possible example includes the CV1 (effective vibration level) which represents "undesired system energy" and causes wear. Therefore the CV1-level should be observed. The number and range of MS will gradually improve over time for a given machine tool. Therefore more and more clusters arise. Some of these clusters represent high wear-proceeding defined by high CV1-levels and caused by higher-thanaverage bearing temperatures while others won't. Consequently machining operations as well as manufactured parts can be categorized and evaluated regarding their wearprocessing characteristics. While some correlations may state the obvious - e.g. heavy machining - the overall load-wear correlation of the machine tool becomes more transparent. Furthermore measurements like switching of an axis position for high wear-processing manufactured parts became practicable.
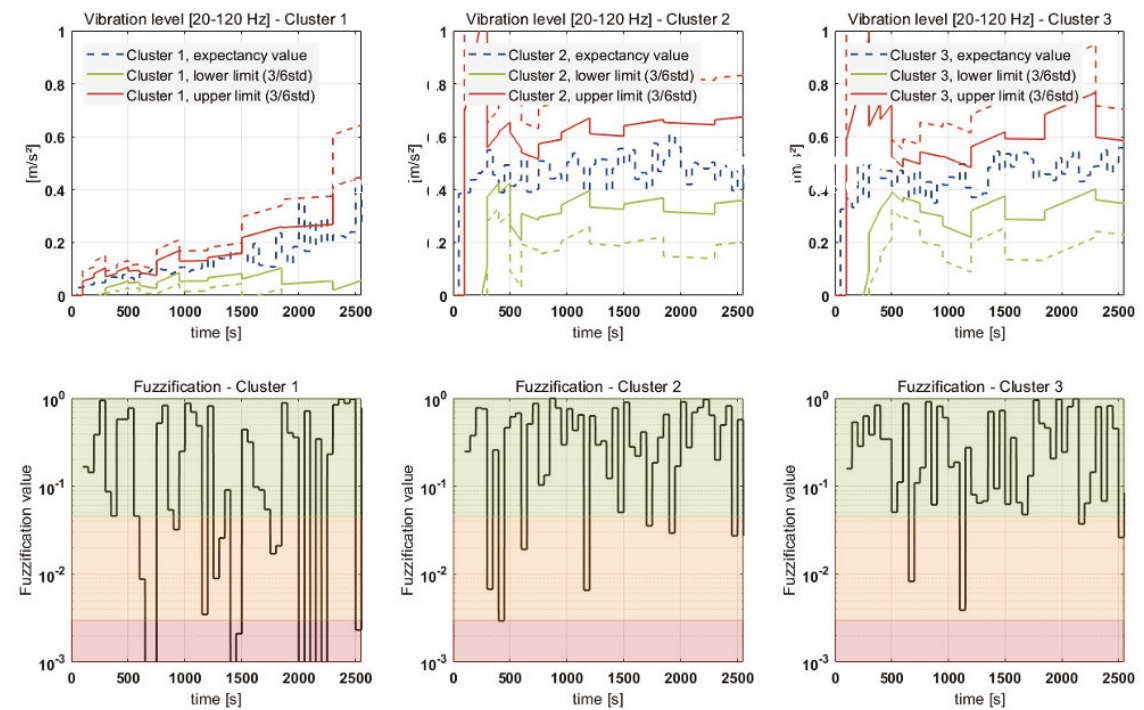

Fig. 5. Cluster-CV progress including Fuzzification ; CV1: Peff of ball-screw nut of Y2 axis

\section{Conclusion}

The auto-definition of relevant MS is crucial for addressing the ongoing changes in internal and external conditions of large and special purpose machine tools. By using a linear regression a significant reduction on the number of MS is possible. This includes the distinction between relevant and irrelevant sub-phases. Furthermore the regression analysis also enables to reduce the number of relevant input TPs (e.g. measuring parameters) per $\mathrm{CV}$.

Based on a subsequent clustering of the machine states these clusters represent a more stable base than a single MS. Their specific TP-ranges in context of specific CVs (e.g. a ball-pass frequency) represent machine tool characteristics. A categorization of processes and manufactured parts - regarding their wear-processing as well as 
quality stability - becomes possible when combined with structural information's and a process-evaluation regarding their cluster attribution.

Further research is necessary due to different clustering approaches as well as more complex regression model approaches (e.g. quadratic). Furthermore, the deduction of complex Characteristic values for entire structural components using several CVs based on different algorithms will be investigated.

\section{Acknowledgements}

The research presented in this paper is funded by the European Union (European Social Fund) and by the Free State of Saxony. The authors would like to thank the founders.

\section{References}

1. Lee, J.; Bagheri, B.; Kao, H.-A.: "A Cyber-Physical Systems architecture for Industry 4.0based manufacturing systems", Manufacturing Letters. 18-23 2015.

2. Lu, Y.: Industry 4.0: a survey on technologies, applications and open research issues. Journal of Industrial Information Integration 6, 1-10 (2017)

3. Gausemeier, J.; Klocke, F.: Industrie 4.0 - International Benchmark, Options for the Future and Recommendations for Manufacturing Research, Paderborn 2016.

4. J. T. Farinha, I. Fonseca, R. Oliveira und H. Raposo, „CMMS - An integrated view from maintenance management to on-line condition monitoring," in Proceedings of Maintenance Performance Measurement and Management (MPMM) Conference, Coimbra, Portugal, 2014.

5. R. Teti, K. Jemielniak, G. O'Donnell and D. Dornfeld, „Advanced monitoring of machining operations,“ CIRP Annals - Manufacturing Technology, Nr. 59, pp. 717-739, 2010.

6. W. Derigent, E. Thomas, E. Levrat and B. Iung, „Opportunistic maintenance based on fuzzy modelling of component Proximity,“ CIRP Annals - Manufacturing Technology, Bd. 58, pp. 29-32, 2009.

7. M. Putz, U. Frieß, M. Wabner, A. Friedrich, A. Zander and H. Schlegel, „State-based and self-adapting Algorithm for Condition Monitoring," in 10th CIRP Conference on Intelligent Computation in Manufacturing Engineering - CIRP ICME '16, Ischia, Naples, Italy, 20 - 22 July 2016

8. U. Frieß, M. Kolouch, M. Putz, A. Friedrich and A. Zander: "Fuzzy-clustering of machine states for condition monitoring", CIRP Journal of Manufacturing Science and Technology, Vol. XX, xxx-xxx, 2018.

9. R. Kruse, C. Borgelt, C. Braune, F. Klawonn, C. Moewes und M. Steinbrecher, Computational Intelligence - Eine methodische Einführung in Künstliche Neuronale Netze, Evolutionäre Algorithmen, Fuzzy-Systeme und Bayes-Netze, Wiesbaden: Springer Vieweg, 2. Auflage 2015. 
Open Access This chapter is licensed under the terms of the Creative Commons Attribution 4.0 International License (http://creativecommons.org/licenses/by/4.0/), which permits use, sharing, adaptation, distribution and reproduction in any medium or format, as long as you give appropriate credit to the original author(s) and the source, provide a link to the Creative Commons licence and indicate if changes were made

The images or other third party material in this chapter are included in the chapter's Creative Commons licence, unless indicated otherwise in a credit line to the material. If material is not included in the chapter's Creative Commons licence and your intended use is not permitted by statutory regulation or exceeds the permitted use, you will need to obtain permission directly from the copyright holder.

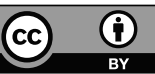

